International Journal of Pure and Applied Mathematics

Volume 114 No. 2 2017, 261-275

ISSN: 1311-8080 (printed version); ISSN: 1314-3395 (on-line version)

url: http://www.ijpam.eu

doi: 10.12732 /ijpam.v114i2.9

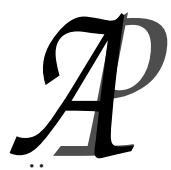

\title{
THE HENSTOCK-STIELTJES INTEGRAL FOR SET-VALUED FUNCTIONS
}

\author{
Muawya Elsheikh Hamid ${ }^{1}$, Luoshan $\mathrm{Xu}^{2}$, Zengtai Gong ${ }^{3}$ \\ ${ }^{1,2}$ School of Mathematical Science \\ Yangzhou University \\ Yangzhou, 225002, P.R. CHINA \\ ${ }^{1}$ School of Management \\ Ahfad University for Women \\ Omdurman, SUDAN \\ ${ }^{3}$ College of Mathematics and Statistics \\ Northwest Normal University \\ Lanzhou, 730070, P.R. CHINA
}

\begin{abstract}
This paper introduces the concept of Henstock-Stieltjes $(H S)$ integrability for set-valued functions and investigates some properties.
\end{abstract}

AMS Subject Classification: 28B20, 26E25, 54C60, 49J53

Key Words: set-valued functions, Henstock-Stieltjes (HS) integral for set-valued functions, support functions $\sigma(x, F(t))$

\section{Introduction}

The calculus of set-valued functions was found to be terribly applicable in many mathematical fields, especially in mathematical economics science, control theory, and statistics. Accordingly, several recent papers deal with the fundamental theory of integration of set-valued functions, and a number of other approaches were established.

Received: December 12, 2016

Revised: $\quad$ February 25, 2017

Published: $\quad$ May 6, 2017

(c) 2017 Academic Publications, Ltd. url: www.acadpubl.eu

${ }^{\S}$ Correspondence author 
One approach was developed by Aumann. Aumann in [1] introduced the definition of set-valued integral. This concept was supported on the Lebesgue integral for choices of set-valued function. A second approach was employed by Debrue [4], who used an embedding of the convex-compact sets into a Banach space and then considered the Bochner integral into the Banach space. In the both approaches outlined above, the main tools were measure theoretic techniques. In 2014, Ratno [11] presented a characterizations of the HenstockKurzweil integral for a more general class of set-valued functions in Banach spaces which have a finite dimension. He compares the results of the HenstockKurzweil integral with Debrue [4] and Aumann [1] ones.

In the classical real analysis, as a natural extension for Bernhard Riemann integral and Lebesgue integral, the Stieltjes integral plays a very important role in probability theory, random processes, physics, economics science, biometrics and numerical analysis $[2,3,5]$.

In 1998, Gwang et al. [6] defined the Henstock-Stieltjes integral for realvalued functions and proved some properties. In 2007, Hai et al. [8] defined and discussed the Henstock integral of set-valued functions. A necessary and sufficient condition of $(H I)$ integrability for set-valued functions is given by means that of real functions. The main purpose of the present paper is to apply the Henstock-Stieltjes integral to set-valued functions.

The paper is organized as follows, in Section 2 we shall review the relevant concepts and properties of set-valued functions and the definition of HenstockStieltjes integrability for real valued functions. Section 3 is devoted to discussing Henstock-Stieltjes integral for set-valued functions. In Section 4, we introduce some linearity properties of Henstock-Stieltjes integrability for set-valued functions. The last section provides Conclusions.

\section{Preliminaries}

In this paper the close interval $[a, b]$ denotes a compact interval on $\mathrm{R}$. The set of intervals-point $\left\{\left(\left[a_{1}, b_{1}\right], \xi_{1}\right),\left(\left[a_{2}, b_{2}\right], \xi_{2}\right), \cdots,\left(\left[a_{k}, b_{k}\right], \xi_{k}\right)\right\}$ is called a division of $[a, b]$ that is $\xi_{1}, \xi_{2}, \cdots, \xi_{k} \in[a, b]$, intervals $\left[a_{1}, b_{1}\right],\left[a_{2}, b_{2}\right], \cdots,\left[a_{k}, b_{k}\right]$ are non-intersect and $\bigcup_{i=1}^{k}\left[a_{i}, b_{i}\right]=[a, b]$. Marking the division of $[a, b]$ as $P=$ $\left\{\left(\left[a_{1}, b_{1}\right], \xi_{1}\right),\left(\left[a_{2}, b_{2}\right], \xi_{2}\right), \cdots,\left(\left[a_{k}, b_{k}\right], \xi_{k}\right)\right\}$, shortening as $P=\{[u, v] ; \xi\}[10]$.

Definition 2.1. $[9,10]$ Let $P$ be a division of $[a, b]$, if there exists function $\delta>0$ on $[a, b]$, such that $\xi_{i} \in\left[a_{i}, b_{i}\right] \subset\left(\xi_{i}-\delta\left(\xi_{i}\right), \xi_{i}+\delta\left(\xi_{i}\right)\right)(i=1,2, \cdots, k)$, $P$ is said to be a $\delta$ - fine division of $[a, b]$. 
Definition 2.2. [6] Let $\alpha:[a, b] \rightarrow \mathbf{R}$ be an increasing function. A real function $F:[a, b] \rightarrow R$ is Henstock-Stieltjes (or $R H S$ ) integrable to $I \in R$ with respect to $\alpha$ on $[a, b]$ if for every $\varepsilon>0$, there is a function $\delta(t)>0$, such that for any $\delta$ - fine division $P=\left\{\left[u_{i}, v_{i}\right] ; \xi_{i}\right\}_{i=1}^{n}$ we have

$$
\left|\sum_{i=1}^{n} F\left(\xi_{i}\right)\left[\alpha\left(v_{i}\right)-\alpha\left(u_{i}\right)\right]-I\right|<\varepsilon
$$

We write $(R H S) \int_{a}^{b} F(t) \mathrm{d} \alpha=I$, and $F \in H S_{\alpha}[a, b]$.

The symbol $P_{k}\left(R^{n}\right)$ denotes the family of all nonempty compact convex subsets of $R^{n}$, define the addition and scalar multiplication in $P_{k}\left(R^{n}\right)$ as following, for $A, B \in P_{k}\left(R^{n}\right), a \in R$,

$$
A+B=\{x+y \mid x \in A, y \in B\}, \quad a A=\{a x \mid x \in A\} .
$$

For every $A, B \in P_{k}\left(R^{n}\right)$, define the Hausdorff metric of $A$ and $B$ by the equation $[12]$

$$
d(A, B)=\max \left\{\sup _{a \in A} \inf _{b \in B}\|a-b\|, \sup _{b \in B} \inf _{a \in A}\|b-a\|\right\} .
$$

Definition 2.3. [8] The set-valued function $F:[a, b] \rightarrow P_{k}\left(R^{n}\right)$ is said to be Henstock (or $H$ ) integrable on $[a, b]$, if there exists $A \in P_{k}\left(R^{n}\right)$, for every $\varepsilon>0$, there is a function $\delta(t)>0$, such that for any $\delta$ - fine division $P=\{[u, v] ; \xi\}$ of $[a, b]$, we have

$$
d\left(\sum F(\xi)(v-u), A\right)<\varepsilon
$$

Write $(H) \int_{a}^{b} F(t) \mathrm{d} t=A$.

Definition 2.4. [8] For $A \in P_{k}\left(R^{n}\right), x \in S^{n-1}$, define the support function of $A$ as $\sigma(x, A)=\sup _{y \in A}\langle y, x\rangle$, where $S^{n-1}$ is the unit sphere of $R^{n}$, i.e., $S^{n-1}=$ $\left\{x \in R^{n}:\|x\|=1\right\},\langle\cdot, \cdot\rangle$ is the inner product in $R^{n}$.

Remark 2.1. [8] It is clear, for $A, B \in P_{k}\left(R^{n}\right), x \in S^{n-1}$ :

(1) if $k \geq 0, \sigma(x, k A)=k \sigma(x, A)$;

(2) $\sigma(x, A+B)=\sigma(x, A)+\sigma(x, B)$.

Lemma 2.1. [7] If $A \in P_{k}\left(R^{n}\right), x \in S^{n-1}$, then $A=\left\{y \in R^{n} \mid\langle y, x\rangle \leq\right.$ $\left.\sigma(x, A), x \in S^{n-1}\right\}$. 
Lemma 2.2. [7] If $A, B \in P_{k}\left(R^{n}\right)$, then $d(A, B)=\sup _{x \in S^{n-1}} \mid \sigma(x, A)-$ $\sigma(x, B) \mid$.

\section{The Henstock-Stieltjes integral for set-valued functions}

In this section, we shall give the definition of the Henstock-Stieltjes integral for set-valued functions. The basic properties of this integral are discussed by means of the Henstock-Stieltjes integral for real-valued functions and the Henstock integral of set-valued functions.

Definition 3.1. Let $\alpha:[a, b] \rightarrow \mathbf{R}$ be an increasing function. The setvalued function $F:[a, b] \rightarrow P_{k}\left(R^{n}\right)$ is said to be set Henstock-Stieltjes (or $S H S$ ) integrable with respect to $\alpha$ on $[a, b]$, if there exists $I \in P_{k}\left(R^{n}\right)$, for every $\varepsilon>0$, there is a function $\delta(t)>0$, such that for any $\delta$ - fine division $P=\{[u, v], \xi\}$ of $[a, b]$ we have

$$
d\left(\sum_{(P)} F(\xi)[\alpha(v)-\alpha(u)], I\right)<\varepsilon .
$$

We write $(S H S) \int_{a}^{b} F(t) \mathrm{d} \alpha=I$.

Remark 3.1. Let $\alpha:[a, b] \rightarrow \mathbf{R}$ be an increasing function. If the setvalued function $F:[a, b] \rightarrow P_{k}\left(R^{n}\right)$ is $(S H S)$ integrable with respect to $\alpha$ on $[a, b]$, then there exists a unique integral value.

Theorem 3.1. Let $\alpha:[a, b] \rightarrow \boldsymbol{R}$ be an increasing function. The setvalued function $F:[a, b] \rightarrow P_{k}\left(R^{n}\right)$ is $(S H S)$ integrable with respect to $\alpha$ on $[a, b]$ if and only if $\sigma(x, F(t))$ is (RHS) integrable on $[a, b]$ uniformly for $x \in S^{n-1}$, and for any $x \in S^{n-1}$, we have

$$
\sigma\left(x,(S H S) \int_{a}^{b} F(t) d \alpha\right)=(R H S) \int_{a}^{b} \sigma(x, F(t)) d \alpha .
$$

Proof. Since set-valued function $F:[a, b] \rightarrow P_{k}\left(R^{n}\right)$ is $(S H S)$ integrable with respect to $\alpha$ on $[a, b]$, then there exists $I \in P_{k}\left(R^{n}\right)$, given $\varepsilon>0$, there is $\delta(t)>0$, for any $\delta$ - fine division $P=\{[u, v], \xi\}$ of $[a, b]$, we have

$$
d\left(\sum_{(P)} F(\xi)[\alpha(v)-\alpha(u)], I\right)<\varepsilon .
$$


By Remark 2.1 and Lemma 2.2,

$$
\begin{aligned}
& \sup _{x \in S^{n-1}}\left|\sum_{(P)} \sigma(x, F(\xi))[\alpha(v)-\alpha(u)]-\sigma(x, I)\right| \\
= & \sup _{x \in S^{n-1}}\left|\sigma\left(x, \sum_{(P)} F(\xi)[\alpha(v)-\alpha(u)]\right)-\sigma(x, I)\right| \\
= & d\left(\sum_{(P)} F(\xi)[\alpha(v)-\alpha(u)], I\right)<\varepsilon,
\end{aligned}
$$

this prove that $\sigma(x, F(t))$ is $(R H S)$ integrable uniformly for any $x \in S^{n-1}$.

Conversely, since $\sigma(x, F(t))$ is $(R H S)$ integrable with respect to $\alpha$ on $[a, b]$ uniformly for any $x \in S^{n-1}$, then there exists $A_{x} \in R$, given $\varepsilon>0$, there is $\delta(t)>0$, for any $\delta$ - fine division $P=\{[u, v], \xi\}$ of $[a, b]$ and for any $x \in S^{n-1}$,

$$
\left|\sum_{(P)} \sigma(x, F(\xi))[\alpha(v)-\alpha(u)]-A_{x}\right|<\varepsilon .
$$

We can proof $\left\{y \in R^{n} \mid\langle y, x\rangle \leq A_{x}, x \in S^{n-1}\right\} \in P_{k}\left(R^{n}\right)$. It is clear that $\left\{y \in R^{n} \mid\langle y, x\rangle \leq A_{x}, x \in S^{n-1}\right\}$ is nonempty set. Let $a, b \in\left\{y \in R^{n} \mid\langle y, x\rangle \leq\right.$ $\left.A_{x}, x \in S^{n-1}\right\}$, then for any $x \in S^{n-1}$,

$$
\langle a, x\rangle \leq A_{x}, \quad\langle b, x\rangle \leq A_{x}
$$

so for any $s \in[0,1],\langle s a+(1-s) b, x\rangle=s\langle a, x\rangle+(1-s)\langle b, x\rangle \leq A_{x}$, such that $\left\{y \in R^{n} \mid\langle y, x\rangle \leq A_{x}, x \in S^{n-1}\right\}$ is the convex subsets of $R^{n}$. Let $\left\{a_{m}\right\}_{m=1}^{\infty} \subset$ $\left\{y \in R^{n} \mid\langle y, x\rangle \leq A_{x}, x \in S^{n-1}\right\}$, then for each $x \in S^{n-1},\left\langle a_{m}, x\right\rangle \leq A_{x}=$ $(R H S) \int_{a}^{b} \sigma(x, F(t)) \mathrm{d} \alpha$, i.e. $\left\langle a_{m}, x\right\rangle$ is bounded. So for any $x \in S^{n-1},\left\langle a_{m}, x\right\rangle$ there exist convergent subsequence $\left\langle a_{m_{k}}, x\right\rangle$. Let $\lim _{k \rightarrow \infty}\left\langle a_{m_{k}}, x\right\rangle=b_{x}$, then $b_{x} \leq A_{x}$, apparently,

$$
\left\langle\lim _{k \rightarrow \infty} a_{m_{k}}, x\right\rangle=\lim _{k \rightarrow \infty}\left\langle a_{m_{k}}, x\right\rangle=b_{x} \leq A_{x},
$$

i.e. $\lim _{k \rightarrow \infty} a_{m_{k}} \in\left\{y \in R^{n} \mid\langle y, x\rangle \leq A_{x}, x \in S^{n-1}\right\}$. So, $\left\{y \in R^{n} \mid\langle y, x\rangle \leq\right.$ $\left.A_{x}, x \in S^{n-1}\right\}$ is a compact set on $R^{n}$.

Let $\left\{y \in R^{n} \mid\langle y, x\rangle \leq A_{x}, x \in S^{n-1}\right\}=A \in P_{k}\left(R^{n}\right)$. By the Lemma 2.1, $\sigma(x, A)=A_{x}$. So, by Lemma 2.2 and the Remark 2.1, we have,

$$
d\left(\sum_{(P)} F(\xi)[\alpha(v)-\alpha(u)], A\right)
$$




$$
\begin{aligned}
& =\sup _{x \in S^{n-1}}\left|\sigma\left(x, \sum_{(P)} F(\xi)[\alpha(v)-\alpha(u)]\right)-\sigma(x, A)\right| \\
& =\sup _{x \in S^{n-1}}\left|\sum_{(P)} \sigma(x, F(\xi))[\alpha(v)-\alpha(u)]-A_{x}\right| \leq \varepsilon .
\end{aligned}
$$

Theorem 3.2. Let $\alpha:[a, b] \rightarrow \boldsymbol{R}$ be an increasing function such that $\alpha \in C^{1}[a, b]$ and the set-valued function $F=\theta$ almost everywhere on $[a, b]$ then $(F, \alpha) \in S H S[a, b]$ and $\int_{a}^{b} F(t) d \alpha=\theta$. Here $\theta=(0,0,0, \cdots, 0)$ and $\sigma(x, \theta)=$ $\sup _{y \in \theta}\langle y, x\rangle=0$.

Proof. If $F:[a, b] \rightarrow P_{k}\left(R^{n}\right)$ is $(S H S)$ integrable with respect to $\alpha$ on $[a, b]$, then $\sigma(x, F(t))$ is $(R H S)$ integrable on $[a, b]$ uniformly for any $x \in S^{n-1}$. Since $\alpha \in C^{1}[a, b]$, there exists $G>0$ such that $\left|\alpha^{\prime}(t)\right| \leq G$, for all $t \in[a, b]$. By the mean value theorem, there exists $\xi_{i} \in\left[u_{i}, v_{i}\right]$ such that

$$
\alpha\left(v_{i}\right)-\alpha\left(u_{i}\right)=\alpha^{\prime}\left(\xi_{i}\right)\left(v_{i}-u_{i}\right) .
$$

Let $E=\{t \mid F \neq \theta\}$ and for every positive integer $n$, set $E=\bigcup E_{n} \subset[a, b]$, where $E_{n}=\{t|n-1 \leq| \sigma(x, F(t)) \mid<n\}, n=1,2, \cdots$ for each $\varepsilon>0$ and a positive integer $n$, choose an open set $W_{n}$ such that $E_{n} \subset W_{n}$ and $\mu\left(W_{n}\right)<$ $\frac{\epsilon}{n G 2^{n}}$. Define a positive function $\delta$ by

$$
\delta(t)= \begin{cases}1, & t \in[a, b] \backslash E, \\ \delta(t), & \text { such that }(t-\delta(t), t+\delta(t)) \subset W_{n}, t \in E_{n}, n=1,2, \cdots\end{cases}
$$

For any $\delta$ - fine division $P=\left\{\left[u_{i}, v_{i}\right], \xi_{i}\right\}$ we have

$$
\begin{aligned}
\left|\sum \sigma(x, F(\xi))[\alpha(v)-\alpha(u)]\right| & =\sum_{n=1}^{\infty}\left|\sum_{\xi_{n i} \in E_{n}} \sigma\left(x, F\left(\xi_{n i}\right)\right)\left[\alpha\left(v_{n i}\right)-\alpha\left(u_{n i}\right)\right]\right| \\
& =\sum_{n=1}^{\infty}\left|\sum_{\xi_{n i} \in E_{n}} \sigma\left(x, F\left(\xi_{n i}\right)\right) \alpha^{\prime}\left(\xi_{n i}^{\prime}\right)\left(v_{n i}-u_{n i}\right)\right| \\
& <\sum_{n=1}^{\infty} n G \mu\left(W_{n}\right) \\
& <\sum_{n=1}^{\infty} \varepsilon 2^{-n} \\
& =\varepsilon .
\end{aligned}
$$


Theorem 3.3. Let $\alpha:[a, b] \rightarrow \boldsymbol{R}$ be an increasing function. A function $F:[a, b] \rightarrow P_{k}\left(R^{n}\right)$ is (SHS) integrable with respect to $\alpha$ on $[a, b]$ if and only if for every $\varepsilon>0$, there is a function $\delta(t)>0$, such that for any two $\delta$ - fine divisions $P_{1}=\left\{\left[u_{1}, v_{1}\right], \xi_{1}\right\}$ and $P_{2}=\left\{\left[u_{2}, v_{2}\right], \xi_{2}\right\}$, we have

$$
d\left(\sum_{\left(P_{1}\right)} F\left(\xi_{1}\right)\left[\alpha\left(v_{1}\right)-\alpha\left(u_{1}\right)\right], \sum_{\left(P_{2}\right)} F\left(\xi_{2}\right)\left[\alpha\left(v_{2}\right)-\alpha\left(u_{2}\right)\right]\right)<\varepsilon .
$$

Proof. If $F:[a, b] \rightarrow P_{k}\left(R^{n}\right)$ is $(S H S)$ integrable with respect to $\alpha$ on $[a, b]$, then $\sigma(x, F(t))$ is $(R H S)$ integrable on $[a, b]$ uniformly for any $x \in S^{n-1}$. Suppose first that $F$ is $(S H S)$ integrable with respect to $\alpha$ on $[a, b]$ and $\varepsilon>0$, there is a function $\delta(t)>0$ on $[a, b]$ such that

$$
\begin{aligned}
& \left|\sum_{\left(P_{1}\right)} \sigma\left(x, F\left(\xi_{1}\right)\right)\left[\alpha\left(v_{1}\right)-\alpha\left(u_{1}\right)\right]-(S H S) \int_{a}^{b} \sigma\left(x, F\left(t_{1}\right)\right) \mathrm{d} \alpha\right|<\frac{\varepsilon}{2}, \\
& \left|\sum_{\left(P_{2}\right)} \sigma\left(x, F\left(\xi_{2}\right)\right)\left[\alpha\left(v_{2}\right)-\alpha\left(u_{2}\right)\right]-(S H S) \int_{a}^{b} \sigma\left(x, F\left(t_{2}\right)\right) \mathrm{d} \alpha\right|<\frac{\varepsilon}{2} .
\end{aligned}
$$

Whenever $P_{1}$ and $P_{2}$ are two $\delta$ - fine divisions of $[a, b]$. Then

$$
\begin{aligned}
& \left|\sum_{\left(P_{1}\right)} \sigma\left(x, F\left(\xi_{1}\right)\right)\left[\alpha\left(v_{1}\right)-\alpha\left(u_{1}\right)\right]-\sum_{\left(P_{2}\right)} \sigma\left(x, F\left(\xi_{2}\right)\right)\left[\alpha\left(v_{2}\right)-\alpha\left(u_{2}\right)\right]\right| \\
& \leq\left|\sum_{\left(P_{1}\right)} \sigma\left(x, F\left(\xi_{1}\right)\right)\left[\alpha\left(v_{1}\right)-\alpha\left(u_{1}\right)\right]-(S H S) \int_{a}^{b} \sigma\left(x, F\left(t_{1}\right)\right) \mathrm{d} \alpha\right| \\
& +\left|\sum_{\left(P_{2}\right)} \sigma\left(x, F\left(\xi_{2}\right)\right)\left[\alpha\left(v_{2}\right)-\alpha\left(u_{2}\right)\right]-(S H S) \int_{a}^{b} \sigma\left(x, F\left(t_{2}\right)\right) \mathrm{d} \alpha\right| \\
& \quad<\frac{\varepsilon}{2}+\frac{\varepsilon}{2} \\
& \quad=\varepsilon .
\end{aligned}
$$

Hence, the Cauchy criterion is satisfied.

Conversely, suppose that the Cauchy criterion is satisfied. For each positive integer $n$, choose $\delta_{n}(t)>0$ on $[a, b]$ such that

$$
\left|\sum_{\left(P_{1}\right)} \sigma\left(x, F\left(\xi_{1}\right)\right)\left[\alpha\left(v_{1}\right)-\alpha\left(u_{1}\right)\right]-\sum_{\left(P_{2}\right)} \sigma\left(x, F\left(\xi_{2}\right)\right)\left[\alpha\left(v_{2}\right)-\alpha\left(u_{2}\right)\right]\right|<\frac{1}{n} .
$$


Whenever $P_{1}$ and $P_{2}$ are two $\delta$ - fine divisions of $[a, b]$. We may assume that the sequence $\left\{\delta_{n}(t)\right\}$ is non-increasing. For every $n$, let $P_{n}$ be a $\delta_{n}-$ fine division of $[a, b]$. The sequence $\left\{\sigma\left(x, F\left(\xi_{n}\right)\right)\left[\alpha\left(v_{n}\right)-\alpha\left(u_{n}\right)\right]\right\}$ is a Cauchy sequence since

$$
m>n \geq N
$$

implies

$$
\left|\sum_{\left(P_{m}\right)} \sigma\left(x, F\left(\xi_{m}\right)\right)\left[\alpha\left(v_{m}\right)-\alpha\left(u_{m}\right)\right]-\sum_{\left(P_{n}\right)} \sigma\left(x, F\left(\xi_{n}\right)\right)\left[\alpha\left(v_{n}\right)-\alpha\left(u_{n}\right)\right]\right|<\frac{1}{N}
$$

Let $L$ be the limit of this sequence and $\varepsilon>0$. Choose a positive integer $N$ such that

$$
\frac{1}{N}<\frac{\varepsilon}{2} \text { and }\left|\sum_{\left(P_{n}\right)} \sigma\left(x, F\left(\xi_{n}\right)\right)\left[\alpha\left(v_{n}\right)-\alpha\left(u_{n}\right)\right]-L\right|<\frac{\varepsilon}{2} \text { for all } n \leq N .
$$

Let $P$ be a $\delta_{N}$ - fine division of $[a, b]$ and compute

$$
\begin{aligned}
&\left|\sum_{(P)} \sigma(x, F(\xi))[\alpha(v)-\alpha(u)]-L\right| \leq \mid \sum_{(P)} \sigma(x, F(\xi))[\alpha(v)-\alpha(u)] \\
&-\sum_{\left(P_{N}\right)} \sigma\left(x, F\left(\xi_{N}\right)\right)\left[\alpha\left(v_{N}\right)-\alpha\left(u_{N}\right)\right] \mid \\
&+\left|\sum_{\left(P_{N}\right)} \sigma\left(x, F\left(\xi_{N}\right)\right)\left[\alpha\left(v_{N}\right)-\alpha\left(u_{N}\right)\right]-L\right| \\
&<\frac{1}{N}+\frac{\varepsilon}{2} \\
&<\varepsilon .
\end{aligned}
$$

Hence, the function $F$ is $S H S$ integrable with respect to $\alpha$ on $[a, b]$.

Theorem 3.4. Let $\alpha:[a, b] \rightarrow \boldsymbol{R}$ be an increasing function. Let $F$ : $[a, b] \rightarrow P_{k}\left(R^{n}\right)$. If $F$ is $(S H S)$ integrable with respect to $\alpha$ on $[a, b]$, then $F$ is $(S H S)$ integrable with respect to $\alpha$ on every subinterval of $[a, b]$.

Proof. If $F:[a, b] \rightarrow P_{k}\left(R^{n}\right)$ is $(S H S)$ integrable with respect to $\alpha$ on $[a, b]$, then $\sigma(x, F(t))$ is $(R H S)$ integrable on $[a, b]$ uniformly for any $x \in S^{n-1}$. Let $[c, d] \subseteq[a, b]$ and let $\varepsilon>0$. Choose a positive function $\delta$ on $[a, b]$ such that

$$
\left|\sum_{\left(P_{1}\right)} \sigma\left(x, F\left(\xi_{1}\right)\right)\left[\alpha\left(v_{1}\right)-\alpha\left(u_{1}\right)\right]-\sum_{\left(P_{2}\right)} \sigma\left(x, F\left(\xi_{2}\right)\right)\left[\alpha\left(v_{2}\right)-\alpha\left(u_{2}\right)\right]\right|<\varepsilon .
$$


Whenever $P_{1}$ and $P_{2}$ are two $\delta$ - fine divisions of $[a, b]$. Fix $\delta$ - fine division $P_{a}$ of $[a, c]$ and $P_{b}$ of $[d, b]$. Let $P_{1}^{\prime}$ and $P_{2}^{\prime}$ be $\delta$ - fine divisions of $[c, d]$ and define $P_{1}=P_{a} \cup P_{1}^{\prime} \cup P_{b}$ and $P_{2}=P_{a} \cup P_{2}^{\prime} \cup P_{b}$. Then $P_{1}$ and $P_{2}$ are two $\delta-$ fine divisions of $[a, b]$ and

$$
\begin{aligned}
& \left|\sum_{\left(P_{1}^{\prime}\right)} \sigma\left(x, F\left(\xi_{1}^{\prime}\right)\right)\left[\alpha\left(v_{1}^{\prime}\right)-\alpha\left(u_{1}^{\prime}\right)\right]-\sum_{\left(P_{2}^{\prime}\right)} \sigma\left(x, F\left(\xi_{2}^{\prime}\right)\right)\left[\alpha\left(v_{2}^{\prime}\right)-\alpha\left(u_{2}^{\prime}\right)\right]\right| \\
= & \left|\sum_{\left(P_{1}\right)} \sigma\left(x, F\left(\xi_{1}\right)\right)\left[\alpha\left(v_{1}\right)-\alpha\left(u_{1}\right)\right]-\sum_{\left(P_{2}\right)} \sigma\left(x, F\left(\xi_{2}\right)\right)\left[\alpha\left(v_{2}\right)-\alpha\left(u_{2}\right)\right]\right|<\varepsilon .
\end{aligned}
$$

Hence, the function $F$ is $(S H S)$ integrable with respect to $\alpha$ on every subinterval of $[a, b]$.

\section{Linearity properties of Henstock-Stieltjes integrability for set-valued functions}

In this section, we discuss some linearity properties of Henstock-Stieltjes integrability for set-valued functions.

Definition 4.1. Let $\alpha:[a, b] \rightarrow \mathbf{R}$ be an increasing function. Let $F, G$ : $[a, b] \rightarrow P_{k}\left(R^{n}\right)$ be set-valued functions. We say that $F=G$ almost everywhere on $[a, b]$, if $\sigma(x, F(t))-\sigma(x, G(t))=0$ almost everywhere on $[a, b]$ for any $x \in S^{n-1}$.

Lemma 4.1. [6] Let $\alpha$ be an increasing function on $[a, b]$ and let $f=0$ almost everywhere on $[a, b]$. If $\alpha \in C^{1}[a, b]$, then $f$ is (HS) integrable with respect to $\alpha$ on $[a, b]$ and $\int_{a}^{b} f d \alpha=0$.

Lemma 4.2. [6] Let $\alpha$ be an increasing function on $[a, b]$. Let $f$ and $g$ be (HS) integrable with respect to $\alpha$ on $[a, b]$. Then $f+g$ is $(H S)$ integrable with respect to $\alpha$ on $[a, b]$ and

$$
\int_{a}^{b}(f+g) d \alpha=\int_{a}^{b} f d \alpha+\int_{a}^{b} g d \alpha .
$$

Theorem 4.1. Let $\alpha:[a, b] \rightarrow \boldsymbol{R}$ be an increasing function. Let $F$ : $[a, b] \rightarrow P_{k}\left(R^{n}\right)$ and let $c \in(a, b)$. If $F$ is $(S H S)$ integrable with respect to $\alpha$ 
on each of the intervals $[a, c]$ and $[c, b]$, then $F$ is $(S H S)$ integrable with respect to $\alpha$ on $[a, b]$ and

$$
(S H S) \int_{a}^{b} F(t) d \alpha=(S H S) \int_{a}^{c} F(t) d \alpha+(S H S) \int_{c}^{b} F(t) d \alpha .
$$

Proof. If $F:[a, b] \rightarrow P_{k}\left(R^{n}\right)$ is (SHS) integrable with respect to $\alpha$ on $[a, b]$, then $\sigma(x, F(t))$ is $(R H S)$ integrable on $[a, b]$ uniformly for any $x \in S^{n-1}$. Let $\varepsilon>0$. By hypothesis, there exists a positive function $\delta_{1}$ on $[a, c]$ such that

$$
\left|\sum_{(P)} \sigma(x, F(\xi))[\alpha(v)-\alpha(u)]-\int_{a}^{c} \sigma(x, F(t)) \mathrm{d} \alpha\right|<\frac{\varepsilon}{2} .
$$

Whenever $P$ is $\delta_{1}$ - fine division of $[a, c]$, and there exists a positive function $\delta_{2}$ on $[c, b]$ such that

$$
\left|\sum_{(P)} \sigma(x, F(\xi))[\alpha(v)-\alpha(u)]-\int_{c}^{b} \sigma(x, F(t)) \mathrm{d} \alpha\right|<\frac{\varepsilon}{2} .
$$

Whenever $P$ is $\delta_{2}-$ fine division of $[c, b]$.

Define $\delta$ on $[a, b]$ by

$$
\delta(t)= \begin{cases}\min \left\{\delta_{1}(t), c-t\right\}, & \text { if } a \leq t<c \\ \min \left\{\delta_{1}(c), \delta_{2}(c)\right\}, & \text { if } t=c \\ \min \left\{\delta_{2}(t), t-c\right\}, & \text { if } c<t \leq b\end{cases}
$$

Let $P$ be $\delta$ - fine division of $[a, b]$ that each division occurs only once. Note that $P$ must be of the form $P_{a} \cup(c,[u, v]) \cup P_{b}$ where $\delta$ - fine division $P_{a}$ are less than $c$ and $\delta$ - fine division $P_{b}$ are grater than $c$. Let $P_{1}=P_{a} \cup(c,[u, c])$ and let $P_{2}=P_{b} \cup(c,[c, v])$. Then $P_{1}$ is $\delta_{1}$ - fine division of $[a, c]$ and $P_{2}$ is $\delta_{2}-$ fine division of $[c, b]$.

Since

$$
\begin{aligned}
\left|\sum_{(P)} \sigma(x, F(\xi))[\alpha(v)-\alpha(u)]\right|=\mid & \sum_{\left(P_{1}\right)} \sigma\left(x, F\left(\xi_{1}\right)\right)\left[\alpha\left(v_{1}\right)-\alpha\left(u_{1}\right)\right] \mid \\
& +\left|\sum_{\left(P_{2}\right)} \sigma\left(x, F\left(\xi_{2}\right)\right)\left[\alpha\left(v_{2}\right)-\alpha\left(u_{2}\right)\right]\right| .
\end{aligned}
$$

We obtain 


$$
\begin{aligned}
& \left|\sum_{(P)} \sigma(x, F(\xi))[\alpha(v)-\alpha(u)]-\int_{a}^{c} \sigma(x, F(t)) \mathrm{d} \alpha-\int_{c}^{b} \sigma(x, F(t)) \mathrm{d} \alpha\right| \\
& \leq\left|\sum_{\left(P_{1}\right)} \sigma\left(x, F\left(\xi_{1}\right)\right)\left[\alpha\left(v_{1}\right)-\alpha\left(u_{1}\right)\right]-\int_{a}^{c} \sigma\left(x, F\left(t_{1}\right)\right) \mathrm{d} \alpha\right| \\
& +\left|\sum_{\left(P_{2}\right)} \sigma\left(x, F\left(\xi_{2}\right)\right)\left[\alpha\left(v_{2}\right)-\alpha\left(u_{2}\right)\right]-\int_{c}^{b} \sigma\left(x, F\left(t_{2}\right)\right) \mathrm{d} \alpha\right|<\varepsilon
\end{aligned}
$$

Theorem 4.2. Let $\alpha:[a, b] \rightarrow \boldsymbol{R}$ be an increasing function. Let $F, G$ : $[a, b] \rightarrow P_{k}\left(R^{n}\right)$ be $(S H S)$ integrable with respect to $\alpha$ on $[a, b]$ and $k_{1}, k_{2} \in R$ then

$$
\begin{aligned}
(S H S) \int_{a}^{b}\left(k_{1} F(t)+k_{2} G(t)\right) d \alpha & \\
& =k_{1}(S H S) \int_{a}^{b} F(t) d \alpha+k_{2}(S H S) \int_{a}^{b} G(t) d \alpha .
\end{aligned}
$$

Proof. Let $F, G$ be $(S H S)$ integrable with respect to $\alpha$ on $[a, b]$.

Case $1 . k_{1}=k_{2}=0$, it is obvious;

Case 2. $k_{1}=0, k_{2} \neq 0$, it is obvious;

Case 3. $k_{1} \neq 0, k_{2}=0$, it is obvious;

Case $4 . k_{1} \neq 0, k_{2} \neq 0$.

Let $(S H S) \int_{a}^{b} F(t) \mathrm{d} \alpha=A$ and $(S H S) \int_{a}^{b} G(t) \mathrm{d} \alpha=B . \quad$ Then for every $\varepsilon>0$, there is a function $\delta(t)>0$, such that for any $\delta_{1}-$ fine division $P_{1}=\left\{\left[u_{1}, v_{1}\right], \xi_{1}\right\}$, we have

$$
d\left(\sum_{\left(P_{1}\right)} F\left(\xi_{1}\right)\left[\alpha\left(v_{1}\right)-\alpha\left(u_{1}\right)\right], A\right)<\frac{\varepsilon}{2\left|k_{1}\right|} .
$$

And for any $\delta_{2}-$ fine division $P_{2}=\left\{\left[u_{2}, v_{2}\right], \xi_{2}\right\}$, we have

$$
d\left(\sum_{\left(P_{2}\right)} G\left(\xi_{2}\right)\left[\alpha\left(v_{2}\right)-\alpha\left(u_{2}\right)\right], B\right)<\frac{\varepsilon}{2\left|k_{2}\right|} .
$$

Define $\delta$ on $[a, b]$ by

$$
\delta(t)=\min \left\{\delta\left(t_{1}\right), \delta\left(t_{2}\right)\right\}
$$


Let $P$ be a $\delta$ - fine division of $[a, b]$. Then

$$
\begin{aligned}
& d\left(\sum_{(P)}\left(k_{1} F(\xi)+k_{2} G(\xi)\right)[\alpha(v)-\alpha(u)],\left(k_{1} A+k_{2} B\right)\right) \\
\leq & d\left(\sum_{\left(P_{1}\right)} k_{1} F\left(\xi_{1}\right)\left[\alpha\left(v_{1}\right)-\alpha\left(u_{1}\right)\right], k_{1} A\right)+d\left(\sum_{\left(P_{2}\right)} k_{2} G\left(\xi_{2}\right)\left[\alpha\left(v_{2}\right)-\alpha\left(u_{2}\right)\right], k_{2} B\right) \\
= & \left|k_{1}\right| d\left(\sum_{\left(P_{1}\right)} F\left(\xi_{1}\right)\left[\alpha\left(v_{1}\right)-\alpha\left(u_{1}\right)\right], A\right)+\left|k_{2}\right| d\left(\sum_{\left(P_{2}\right)} G\left(\xi_{2}\right)\left[\alpha\left(v_{2}\right)-\alpha\left(u_{2}\right)\right], B\right)<\varepsilon
\end{aligned}
$$

Theorem 4.3. Let $\alpha:[a, b] \rightarrow \boldsymbol{R}$ be an increasing function. Let $F, G$ : $[a, b] \rightarrow P_{k}\left(R^{n}\right)$ be $(S H S)$ integrable with respect to $\alpha$ on $[a, b]$ and let $\alpha \in$ $C^{1}[a, b]$. If $F=G$ almost everywhere on $[a, b]$, then

$$
(S H S) \int_{a}^{b} F(t) d \alpha=(S H S) \int_{a}^{b} G(t) d \alpha .
$$

Proof. By Definition 4.1, if $F=G$ almost everywhere on $[a, b]$, then $\sigma(x, F(t))-$ $\sigma(x, G(t))=0$ almost everywhere on $[a, b]$ for any $x \in S^{n-1}$. By Theorem 3.1 and Lemma $4.1 \sigma(x, F(t))-\sigma(x, G(t))$ is $(R H S)$ integrable with respect to $\alpha$ on $[a, b]$ and

$$
\int_{a}^{b}(\sigma(x, F(t))-\sigma(x, G(t))) \mathrm{d} \alpha=0 .
$$

By Lemma 4.2 the support function

$$
\sigma(x, G(t))=\sigma(x, F(t))+[\sigma(x, G(t))-\sigma(x, F(t))],
$$

is $(R H S)$ integrable with respect to $\alpha$ on $[a, b]$ and

$$
\begin{aligned}
&(R H S) \int_{a}^{b} \sigma(x, G(t)) \mathrm{d} \alpha \\
&=(R H S) \int_{a}^{b} \sigma(x, F(t)) \mathrm{d} \alpha+(R H S) \int_{a}^{b}[\sigma(x, G(t))-\sigma(x, F(t))] \mathrm{d} \alpha \\
&=(R H S) \int_{a}^{b} \sigma(x, F(t)) \mathrm{d} \alpha .
\end{aligned}
$$


Theorem 4.4. (Saks-Henstock Lemma). Let $\alpha:[a, b] \rightarrow \boldsymbol{R}$ be an increasing function. Let $F:[a, b] \rightarrow P_{k}\left(R^{n}\right)$ be $(S H S)$ integrable with respect to $\alpha$ on $[a, b]$. Then for every $\varepsilon>0$, there is a function $\delta(t)>0$, such that

$$
d\left(\sum_{(P)} F(\xi)[\alpha(v)-\alpha(u)], \int_{a}^{b} F(t) d \alpha\right)<\varepsilon,
$$

for each $\delta$ - fine division $P=\left\{\left[u_{i}, v_{i}\right] ; \xi_{i}\right\}_{i=1}^{n}$ of $[a, b]$. Particulary, if $P^{\prime}=$ $\left\{\left[u_{i}^{\prime}, v_{i}^{\prime}\right] ; \xi_{i}^{\prime}\right\}_{i=1}^{m}$ is an arbitrary $\delta$ - fine partial division of $[a, b]$, we have

$$
d\left(\sum_{\left(P^{\prime}\right)} F\left(\xi^{\prime}\right)\left[\alpha\left(v^{\prime}\right)-\alpha\left(u^{\prime}\right)\right], \sum_{i=1}^{m} \int_{a}^{b} F(t) d \alpha\right) \leq \varepsilon .
$$

Proof. If $F:[a, b] \rightarrow P_{k}\left(R^{n}\right)$ is $(S H S)$ integrable with respect to $\alpha$ on $[a, b]$, then $\sigma(x, F(t))$ is $(R H S)$ integrable on $[a, b]$ uniformly for any $x \in S^{n-1}$. Assume $P^{\prime}=\left\{\left[u_{i}^{\prime}, v_{i}^{\prime}\right] ; \xi_{i}^{\prime}\right\}_{i=1}^{m}$ is an arbitrary $\delta$ - fine partial division of $[a, b]$, then the complement $[a, b] \backslash \bigcup_{i=1}^{m}\left[u_{i}, v_{i}\right]$ can be expressed as a finite collection of closed subintervals and we denote

$$
[a, b] \backslash \bigcup_{i=1}^{m}\left[u_{i}, v_{i}\right]=\bigcup_{j=1}^{k}\left[u_{j}^{\prime}, v_{j}^{\prime}\right]
$$

Let $\eta>0$ be arbitrary. From Theorem 3.4 we know $\int_{u_{j}^{\prime}}^{v_{j}^{\prime}} \sigma(x, F(t)) \mathrm{d} \alpha$ exists for each $j=1,2, \cdots k$, then there exists $\delta_{j}$ on $\left[u_{j}^{\prime}, v_{j}^{\prime}\right]$ such that if $P_{j}$ is a $\delta_{j}-$ fine division of $\left[u_{j}^{\prime}, v_{j}^{\prime}\right]$, then

$$
\left|\sum_{\left(P_{j}\right)} \sigma\left(x, F\left(\xi_{j}\right)\right)\left[\alpha\left(v_{j}\right)-\alpha\left(u_{j}\right)\right]-\int_{u_{j}^{\prime}}^{v_{j}^{\prime}} \sigma(x, F(t)) \mathrm{d} \alpha\right|<\frac{\eta}{k} .
$$

Assume that $\delta_{j} \subset \delta$ for all $t \in[a, b]$. Let $P_{0}=P^{\prime}+P_{1}+P_{2}+\cdots+P_{k}$, obviously $P_{0}$ is $\delta$ - fine division of $[a, b]$, we have

$$
\left|\sum_{\left(P_{0}\right)} \sigma\left(x, F\left(\xi_{0}\right)\right)\left[\alpha\left(v_{0}\right)-\alpha\left(u_{0}\right)\right]-\int_{a}^{b} \sigma(x, F(t)) \mathrm{d} \alpha\right|
$$




$$
\begin{array}{r}
=\mid \sum_{\left(P^{\prime}\right)} \sigma\left(x, F\left(\xi^{\prime}\right)\right)\left[\alpha\left(v^{\prime}\right)-\alpha\left(u^{\prime}\right)\right]+\sum_{j=1}^{k} \sum_{\left(P_{j}\right)} \sigma\left(x, F\left(\xi_{j}\right)\right)\left[\alpha\left(v_{j}\right)-\alpha\left(u_{j}\right)\right] \\
-\int_{a}^{b} \sigma(x, F(t)) \mathrm{d} \alpha \mid<\varepsilon .
\end{array}
$$

Consequently, we obtain

$$
\begin{aligned}
& \left|\sum_{\left(P^{\prime}\right)} \sigma\left(x, F\left(\xi^{\prime}\right)\right)\left[\alpha\left(v^{\prime}\right)-\alpha\left(u^{\prime}\right)\right]-\sum_{i=1}^{m} \int_{u_{i}}^{v_{i}} \sigma(x, F(t)) \mathrm{d} \alpha\right| \\
= & \mid \sum_{\left(P_{0}\right)} \sigma\left(x, F\left(\xi_{0}\right)\right)\left[\alpha\left(v_{0}\right)-\alpha\left(u_{0}\right)\right]-\sum_{j=1}^{k} \sum_{\left(P_{j}\right)} \sigma\left(x, F\left(\xi_{j}\right)\right)\left[\alpha\left(v_{j}\right)-\alpha\left(u_{j}\right)\right] \\
& -\left(\int_{a}^{b} \sigma(x, F(t)) \mathrm{d} \alpha-\sum_{j=1}^{k} \int_{u_{j}^{\prime}}^{v_{j}^{\prime}} \sigma(x, F(t)) \mathrm{d} \alpha\right) \mid \\
\leq & \left|\sum_{\left(P_{0}\right)} \sigma\left(x, F\left(\xi_{0}\right)\right)\left[\alpha\left(v_{0}\right)-\alpha\left(u_{0}\right)\right]-\int_{a}^{b} \sigma(x, F(t)) \mathrm{d} \alpha\right| \\
& +\sum_{j=1}^{k}\left|\sum_{\left(P_{j}\right)} \sigma\left(x, F\left(\xi_{j}\right)\right)\left[\alpha\left(v_{j}\right)-\alpha\left(u_{j}\right)\right]-\int_{u_{j}^{\prime}}^{v_{j}^{\prime}} \sigma(x, F(t)) \mathrm{d} \alpha\right| \\
< & +\frac{k \eta}{k} \\
= & \varepsilon+\eta .
\end{aligned}
$$

\section{Conclusions}

In summary, this article is to use on Henstock-Stieltjes $(H S)$ integral and the Henstock $(H)$ integral of set-valued functions introduced in $[6,8]$ respectively, to define and discuss the $(H S)$ integral of set-valued functions. As we have seen, this kind of integral is an extension of the set-valued Henstock $(S H)$ integral. Furthermore, we have showed some basic properties of the $(S H S)$ integral by means of the $(H S)$ integral for real-valued functions and the $(H)$ integral of set-valued functions. 


\section{References}

[1] R.J. Aumann, Integrals of set-valued functions, J. Math. Anal. Appl. 12(1965), 1-12, doi: 10.1016/0022-247X(65)90049-1.

[2] P. Billingsley, Probability Measures, John Wiley and Sons Inc., New York (1968).

[3] S.S. Dragomir, The unified treatment of trapezoid, Simpson, and Ostrowski type inequality for monotonic mappings and applications, Mathematical and Computer Modelling, 31(2000), 61-70, doi: 10.1016/S0895-7177(00)00046-7.

[4] G. Debrue, Integration of correspondences, Proc. Fifth Berkeley Sympos. Math. Statist. and Probability, 2, No. 1 (1967), 351-372, Univ.California Press, Berkeley, calif.

[5] L. Egghe, Construction of concentration measures for general Lorenz curves using Riemann-Stieltjes integral, Mathematical and Computer Modelling, 35(2002), 1149-1163, doi: 10.1016/S0895-7177(02)00077-8.

[6] S. Jong, H. Ju, S. Gwang, On Henstock-Stieltjes integral, Kangweon-kyungki Math, 6(1998), 87-96.

[7] S.X. Hai, Z.T. Gong, The McShane integral of set-valued function, Fuzzy Systems and Mathematics, 20, No. 6 (2006), 103-107.

[8] S.X. Hai, F.D. Kong, J.S. Chen. The Henstock integral of set-valued functions, International Journal of Pure and Applied Mathematics, 37, No. 4 (2007), 507-511.

[9] P.Y. Lee, Lanzhou Lectures on Henstock Integration, World Scientific, Singapore, 1989.

[10] P.Y. Lee, R. Vyborny, The Integral: An Easy Approach after Kurzweil and Henstock, Cambridge University Press (2000).

[11] Ratno Bagus Edy Wibowo. The Henstock-Kurzweil Integral of Set-valued Function. International Journal of Mathematical Analysis, 8, No. 55 (2014), 2741- 2755, doi: 10.12988/ijma.2014.410332.

[12] Congxin Wu, Ma Ming, Jinxuan Fang, Structure Theory of Fuzzy Analysis, Guizhou Scientific Publication (1994), In Chinese. 
